\title{
Solid-state structures and thermal properties of inclusion complexes of the phenylurea herbicide cycluron with permethylated cyclodextrins
}

\author{
Dyanne L. Cruickshank, Susan A. Bourne and Mino R. Caira* \\ Department of Chemistry, University of Cape Town, Rondebosch 7701, South Africa \\ E-mail: Mino.Caira@uct.ac.za
}

Dedicated to Professor Rita Hoyos de Rossi in recognition of her contributions to the field of cyclodextrin chemistry

\begin{abstract}
New crystalline inclusion complexes between the urea herbicide cycluron (3-cyclooctyl-1,1dimethylurea) and three methylated cyclodextrins (CDs), namely permethylated $\alpha$-CD (TRIMEA), permethylated $\beta$-CD (TRIMEB) and dimethylated $\beta$-CD (DIMEB), were isolated and studied by single crystal X-ray diffraction and thermal analysis. X-ray analysis of (TRIMEA) $)_{2} \cdot$ cycluron $\cdot 7 \mathrm{H}_{2} \mathrm{O} \quad$ 1, TRIMEB cycluron $\cdot 0.9 \mathrm{H}_{2} \mathrm{O} \quad 2$ and DIMEB cycluron $2.9 \mathrm{H}_{2} \mathrm{O} \quad 3$ revealed significantly different modes of guest inclusion in the complex crystals as well as different crystal packing arrangements. Heating crystals of 1-3 resulted in their dehydration followed by loss of the cycluron guest, but the thermal profiles were distinctly different, complex 3 having the highest thermal stability.
\end{abstract}

Keywords: Cycluron, permethylated cyclodextrins, inclusion complexes, single crystal X-ray diffraction, thermal analysis

\section{Introduction}

Cyclodextrins (CDs) have been studied extensively over the last few decades especially in the pharmaceutical, food, cosmetics and biotechnology industries, as well as in the field of analytical chemistry. ${ }^{1}$ Supramolecular bio-organic applications include encapsulation of drug molecules within the hydrophobic cavity of CD molecules, a strategy which has been shown to improve the bioavailability, chemical stability and absorption of many drugs, and to control drug release rate. $^{2}$ The application of native and derivatised CDs to analogous encapsulation of agrochemical molecules such as herbicides and insecticides should have similar effects to those mentioned for drug formulations. The majority of these agrochemicals are highly insoluble in water, highly 
toxic and have reduced stability against chemical and photolytic degradation. If these adverse physical properties could be reduced or eliminated, it would be possible to use smaller amounts of these toxic chemicals with greater efficacy. An authoritative review of such agrochemical applications has appeared in recent years. ${ }^{3}$

The present study relates to the urea herbicide 3-cyclooctyl-1,1-dimethylurea (Figure 1), which has been used to control the growth of broadleaf and grassy weeds in cereals and other crops and is sparingly soluble in water $\left(\sim 1 \mathrm{mg} / \mathrm{mL}\right.$ at $\left.20{ }^{\circ} \mathrm{C}\right) .{ }^{4} \mathrm{~A}$ recent ${ }^{1} \mathrm{H}-\mathrm{NMR}$ and isothermal titration calorimetric study of the interaction between cycluron and the native host compound $\beta$ $\mathrm{CD}$ in aqueous solution at $25{ }^{\circ} \mathrm{C}$ revealed the formation of a $1: 1$ inclusion complex with an association constant of $4753 \mathrm{M}^{-15}$. This definitive evidence for complex formation prompted the present extended investigation of the affinity of cycluron for methylated CDs, in which the main objectives were to use thermal analysis to assess the effect of complexation on the thermal stability of cycluron and X-ray diffraction to establish the geometry of guest inclusion. The derivatised CDs selected for this study were hexakis(2,3,6-tri- $O$-methyl)- $\alpha$-cyclodextrin (TRIMEA), heptakis(2,3,6-tri- $O$-methyl)- $\beta$-cyclodextrin (TRIMEB) and heptakis(2,6-di- $O$ methyl)- $\beta$-cyclodextrin (DIMEB).

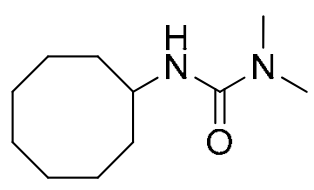

Figure 1. Molecular structure of cycluron.

\section{Results and Discussion}

\section{X-ray diffraction}

Crystal and refinement data for the inclusion complexes are listed in Table 1. Complex 1, (TRIMEA) $)_{2}$ cycluron $7 \mathrm{H}_{2} \mathrm{O}$, contains two host molecules and one guest molecule in the crystal asymmetric unit (Figure 2). The guest molecule is encapsulated in a head-to-head host dimer in which host molecule A accommodates the cyclooctyl residue of the herbicide molecule and host molecule B the amide moiety. Several primary methoxy groups on both host molecules A and B close the narrower side of the host molecule, effecting complete encapsulation of the cycluron molecule. In accommodating very different residues, the host molecules have their secondary faces offset from one another. This is only the second reported occurrence of self-assembly of a head-to-head dimer of TRIMEA encapsulating a guest molecule; our recent account of the interaction between this host and the organothiophosphate insecticide fenitrothion described the inclusion complex (TRIMEA) $)_{2}$ fenitrothion, containing an analogous dimeric motif. ${ }^{6}$ 


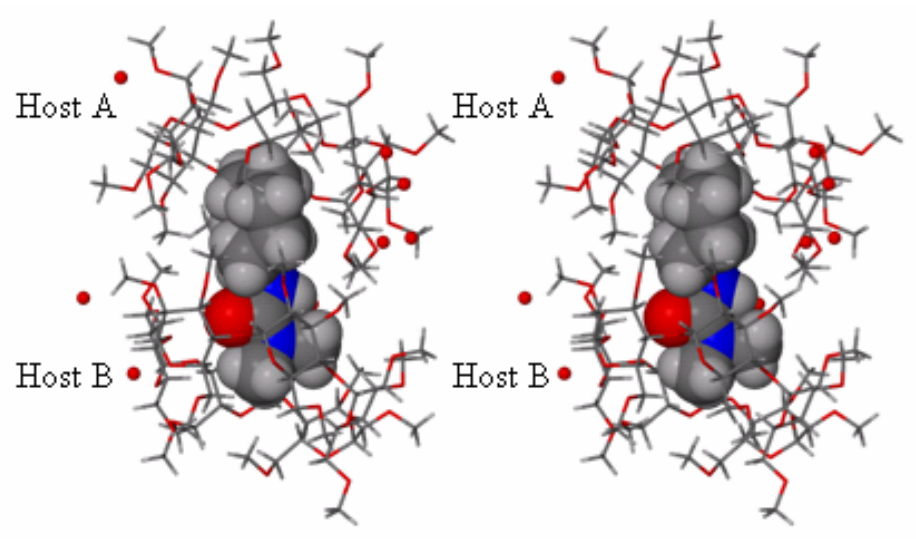

Figure 2. Stereoview of the 2:1 host-guest unit of complex 1, (TRIMEA) $)_{2} \cdot$ cycluron $\cdot 7 \mathrm{H}_{2} \mathrm{O}$.

For the dimeric complex unit, there is a complement of seven water molecules distributed over nine sites. Only one water molecule, with site-occupancy factor (s.o.f.) 0.76, is situated within the dimeric capsule: it serves as a bridge between the guest amide $\mathrm{N}$ atom and a secondary oxygen atom $\mathrm{O} 2$ of the nearest methylated glucose unit of the host, forming a hydrogen bond motif $\mathrm{N} \cdots \mathrm{H}-\mathrm{O}-\mathrm{H} \cdots \mathrm{O} 2$. The hydrogen bond to nitrogen is weak $[\mathrm{N} \cdots \mathrm{O}=3.30(1)$ $\AA]$ while that to $\mathrm{O} 2$ is of average strength $[\mathrm{O} \cdots \mathrm{O}=2.87(1) \AA]$. Interestingly, the potential hydrogen bonding donor ability of the $\mathrm{N}-\mathrm{H}$ group in cycluron is not utilised in the crystal. All the remaining water molecules are either within hydrogen bonding distance of a host oxygen atom or another water molecule. The CD dimers of 1 pack in a modified herringbone formation when viewed down the crystal $c$-axis. The primary (narrower) side of each $\mathrm{CD}$ dimer is blocked by side-on contact with another CD dimer (Figure 3).

Table 1. Crystal data and refinement details for complexes 1, 2 and $3^{\mathrm{a}}$

\begin{tabular}{cccc}
\hline & $\mathrm{H}=$ TRIMEA & $\mathrm{H}=\mathrm{TRIMEB}$ & $\mathrm{H}=$ DIMEB \\
\hline \multirow{2}{*}{ Complex formula } & $(\mathbf{1})\left(\mathrm{C}_{54} \mathrm{H}_{96} \mathrm{O}_{30}\right)_{2} \cdot$ & $(2) \mathrm{C}_{63} \mathrm{H}_{112} \mathrm{O}_{35} \cdot$ & $(3) \mathrm{C}_{56} \mathrm{H}_{98} \mathrm{O}_{35} \cdot$ \\
& $\mathrm{C}_{11} \mathrm{H}_{22} \mathrm{~N}_{2} \mathrm{O} \cdot 7 \mathrm{H}_{2} \mathrm{O}$ & $\mathrm{C}_{11} \mathrm{H}_{22} \mathrm{~N}_{2} \mathrm{O} \cdot 0.9 \mathrm{H}_{2} \mathrm{O}$ & $\mathrm{C}_{11} \mathrm{H}_{22} \mathrm{~N}_{2} \mathrm{O} \cdot 2.9 \mathrm{H}_{2} \mathrm{O}$ \\
$M$ & 2775.08 & 1644.32 & 1582.58 \\
Crystal system & Orthorhombic & Orthorhombic & Orthorhombic \\
Space group & $\mathrm{P} 2{ }_{1} 2_{1} 2_{1}$ & $\mathrm{P} 2{ }_{1} 2{ }_{1} 2_{1}$ & $\mathrm{P} 2{ }_{1} 2{ }_{1} 2_{1}$ \\
$a / \AA$ & $17.1400(3)$ & $14.8872(4)$ & $10.387(1)$ \\
$b / \AA$ & $28.2883(4)$ & $21.2437(6)$ & $15.092(1)$ \\
$c / \AA$ & $30.0478(5)$ & $27.9334(5)$ & $51.862(4)$ \\
$V_{\text {cell }} / \AA^{3}$ & $14569.0(4)$ & $8834.3(4)$ & $8130(1)$ \\
$\mathrm{Z}$ & 4 & 4 & 4 \\
$T / \mathrm{K}$ & $173(2)$ & $173(2)$ & $143(2)$ \\
$\mathrm{F}(000)$ & 6000 & 3556 & 3413 \\
Theta range & $1.0-25.0$ & $2.4-25.0$ & $2.7-24.9$ \\
\hline
\end{tabular}


Table 1. Continued

\begin{tabular}{cccc}
\hline & $\mathrm{H}=$ TRIMEA & $\mathrm{H}=$ TRIMEB & $\mathrm{H}=\mathrm{DIMEB}$ \\
\hline Index ranges & $-20 \leq \mathrm{h} \leq 20$, & $-17 \leq \mathrm{h} \leq 17$, & $-12 \leq \mathrm{h} \leq 8$, \\
& $-33 \leq \mathrm{k} \leq 33$, & $-25 \leq \mathrm{k} \leq 25$, & $-17 \leq \mathrm{k} \leq 17$, \\
& $-35 \leq 1 \leq 35$ & $-33 \leq 1 \leq 33$ & $-61 \leq 1 \leq 56$ \\
Reflections collected & 25686 & 15583 & 45501 \\
$\begin{array}{c}\text { Observed reflections } \\
{[\mathrm{I}>2 \sigma(\mathrm{I})]}\end{array}$ & 13851 & 8496 & 7718 \\
$\begin{array}{c}\text { Data/parameters } \\
\text { Goodness-of-fit on } \mathrm{F}^{2}\end{array}$ & $25686 / 1645$ & $15583 / 988$ & $45501 / 858$ \\
Final $\mathrm{R}$ indices, & 1.017 & 1.048 & 1.298 \\
{$[\mathrm{I}>2 \sigma(\mathrm{I})]$} & $\mathrm{R}_{1}=0.0677$, & $\mathrm{R}_{1}=0.0801$, & $\mathrm{R}_{1}=0.1118$, \\
Largest diff. peak and & $\mathrm{wR}_{2}=0.1990$ & $\mathrm{wR}_{2}=0.2137$ & $\mathrm{wR}_{2}=0.3049$ \\
hole $\left(\mathrm{e} \AA^{-3}\right)$ & $0.66,-0.42$ & $0.65,-0.54$ & $0.66,-0.53$ \\
\hline
\end{tabular}

${ }^{a}$ Full crystallographic data (1. CCDC 783624, 2. CCDC 783625 and 3. CCDC 783623)

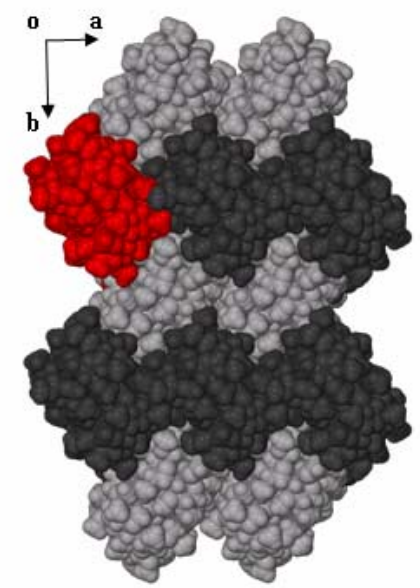

Figure 3. Space-filling representation of the molecular packing in complex 1 viewed along [001]. Different shading/colour is used to distinguish the symmetry-related dimeric capsules.

In contrast to complex 1 , TRIMEB cycluron $0.9 \mathrm{H}_{2} \mathrm{O} 2$ is monomeric. A space-filling diagram displays how the guest molecule is almost totally included within the host cavity, with only the dimethyl urea 'tail' protruding slightly from the secondary side of the CD [Figure 4(a)]. The geometrical parameters of the host are very similar to those reported for isostructural CD complexes. ${ }^{6,7}$ There are two water molecule sites, $\mathrm{O} 1 \mathrm{~W}$ and $\mathrm{O} 2 \mathrm{~W}$, with s.o.f.s 0.44 and 0.47 , respectively. These disordered water molecules are hydrogen bonded to one another $[\mathrm{O} 1 \mathrm{~W} \cdots \mathrm{O} 2 \mathrm{~W} 2.88(2) \AA]$. O1W in turn is a H-bond donor to both the $\mathrm{N}$ atom of the guest molecule $[\mathrm{O} 1 \mathrm{~W} \cdots \mathrm{N} 2.82(2) \AA]$ and a methoxyl oxygen atom $\mathrm{O} 2$ of a methylglucose unit $(\mathrm{O} \cdots \mathrm{O}$ 
2.96(2) $\AA$ ). Finally, the terminal O2W donates one H-bond to a pyranose oxygen atom O5 of a

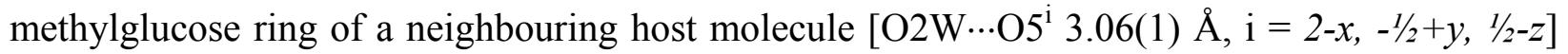
and a second H-bond to a methylglucose $\mathrm{O} 6$ atom of a third host molecule [O2W $\cdots 6^{\mathrm{ii}} 2.83$ (2) $\AA, \mathrm{ii}=2-x,-1 / 2+y, 1 / 2-z]$. As in complex 1 above, the hydrogen atom of the N-H functionality does not appear to engage in hydrogen bonding. Intramolecular and intermolecular $\mathrm{C}-\mathrm{H} \cdots \mathrm{O}$ hydrogen bonds also contribute to stabilization of the crystal structure. Molecules of complex 2 pack in a screw-channel mode in head-to-tail fashion. An undulating, discontinuous host 'channel' is generated parallel to the crystal $b$-axis but guest molecules are isolated from one another. A projection of the complex packing arrangement down [100] is shown in Figure 4(b).
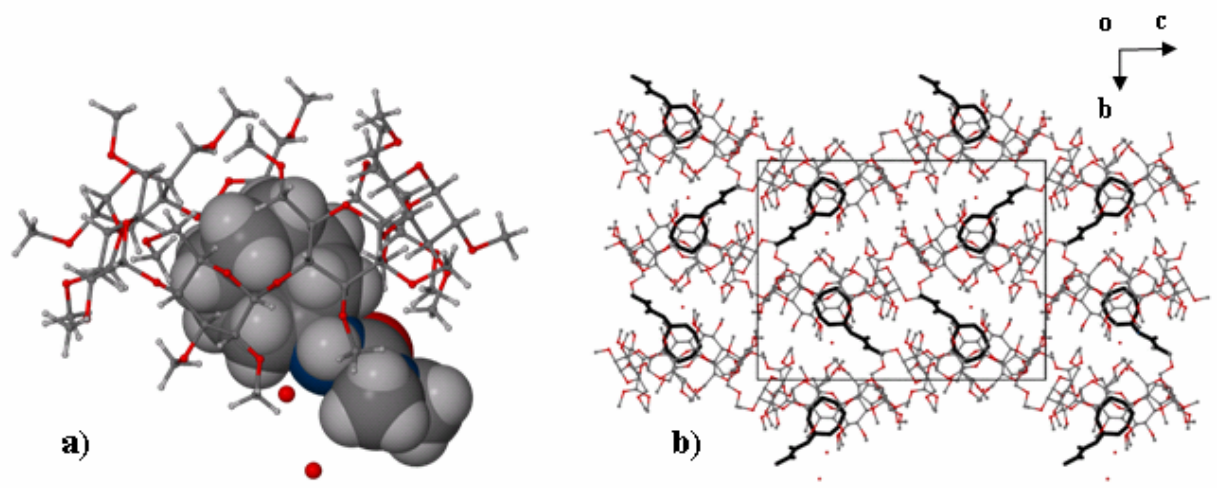

Figure 4. Molecular structure of complex 2 (a); the packing arrangement in 2 viewed down [100] (b).

In the final complex in this series, DIMEB cycluron $2.9 \mathrm{H}_{2} \mathrm{O} \quad 3$, the crystallographic asymmetric unit comprises a hydrated monomeric complex (Figure 5a), but in this case the guest molecule is disordered over two positions. The disordered guest component A has s.o.f. 0.59 while that of component B is 0.41 (Figure $5 b$ ). Both disordered guest components are located in the $\mathrm{CD}$ cavity in the opposite orientation to that found in complex 2 , the hydrophobic cyclooctyl residue now being positioned at the secondary rim of the $\mathrm{CD}$ while the dimethyl urea moiety is located at the narrower primary side. The DIMEB molecule adopts its usual round and symmetrical structure which is maintained by the well-known intramolecular $\mathrm{O} 2(n) \cdots \mathrm{O} 3(n-1)$ hydrogen bonds [distance range 2.79(1)-2.94(1) $\AA$ ] that link contiguous methylglucose residues. This contributes significantly to the rigidity of the macrocycle. The 2.9 water molecules per complex unit are disordered, occupying five extra-cavity sites. Intermolecular hydrogen bonds form between these partially occupied water molecules and between the water molecules and the host oxygen atoms. 

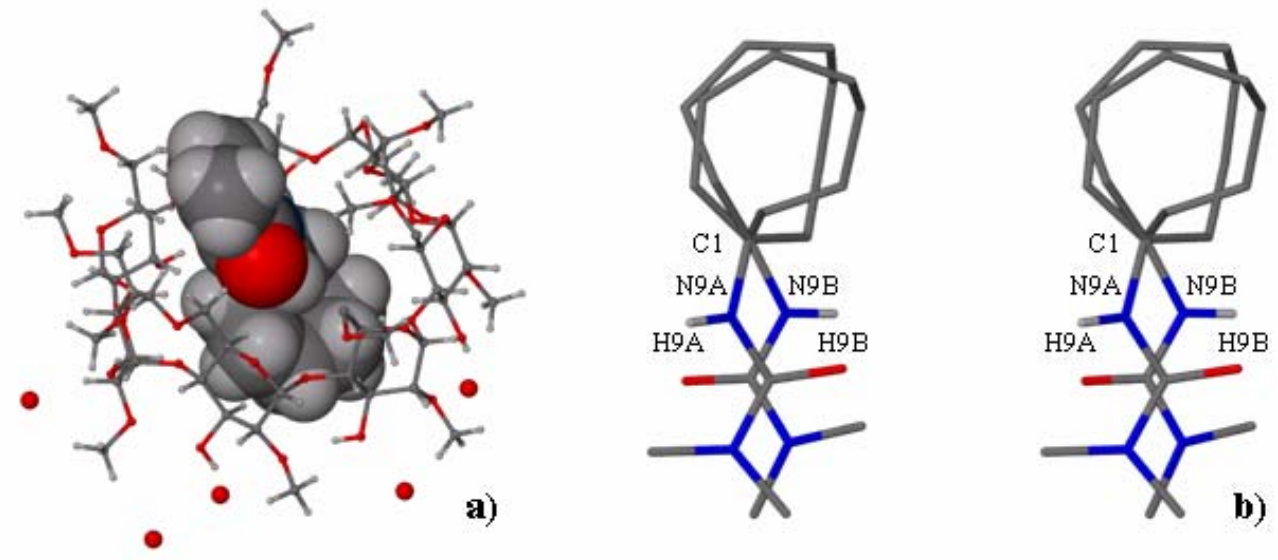

Figure 5. Molecular structure of complex 3 viewed from the host primary side (a); a stereo- view of the disordered guest molecules in complex 3 (for clarity only hydrogen atoms H9A and H9B are included) (b).

The DIMEB complex packs in head-to-tail fashion forming infinite columns along the crystal $a$-axis. A zigzag layer pattern is evident in the [010] projection (Figure 6). Because of the strong inclination of the macrocyclic O4-plane to the $a$-axis direction, a given guest molecule is blocked within its $\mathrm{CD}$ cavity by atoms of neighbouring host molecules at the primary and secondary sides.

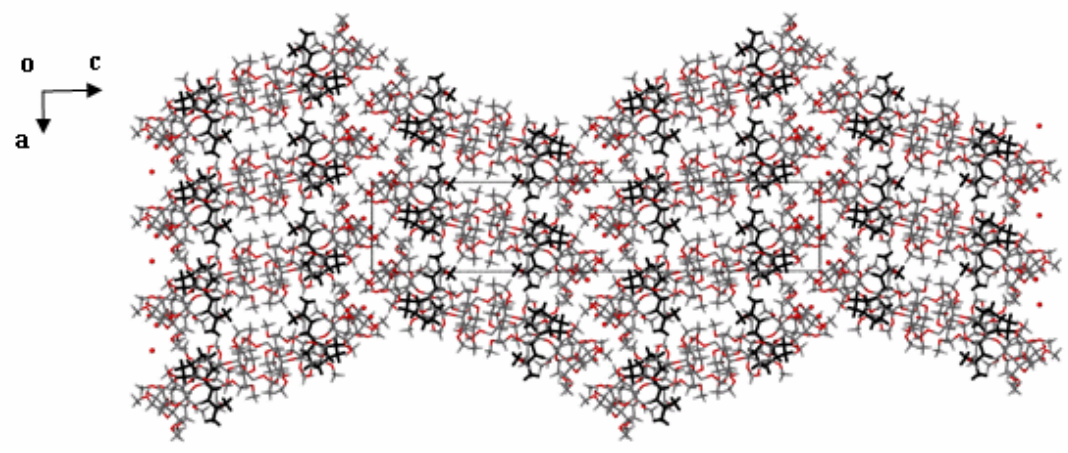

Figure 6. Packing arrangement of complex 3 viewed along [010].

A saturated, eight-membered ring such as that present in cycluron can adopt ten idealised symmetrical conformations. ${ }^{8}$ An overlay of the dimethyl urea residue of the guest molecules occurring in the three complexes shows the various orientations adopted by the cyclooctyl ring (Figure 7). The ring, however, retains a boat-chair conformation (point group $C_{s}$ ) in all cases. 


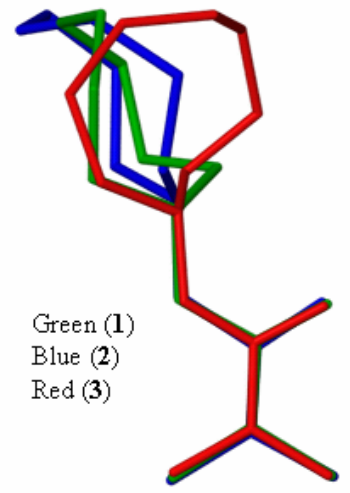

Figure 7. Overlay of the cycluron conformers occurring in the three complexes. (In the case of complex 3, only the major component of guest disorder is shown for simplicity).

\section{Thermal analysis}

Complexes 1-3 are characterized by significant chemical and crystallographic differences which in turn result in distinctly different behaviours upon heating. A summary of the TGA and DSC data and their interpretation for these three complexes follows.

Complex (1). Figure 8(a) illustrates the thermal events recorded for the complex (TRIMEA) $)_{2} \cdot$ cycluron $\cdot 7 \mathrm{H}_{2} \mathrm{O}$. There is a broad endothermic event (A) associated with crystal dehydration in the temperature range $30-100{ }^{\circ} \mathrm{C}$. This corresponds to an observed mass loss in the TGA curve of $4.3 \pm 0.1 \%(\mathrm{n}=2$, yielding $6.5 \pm 0.1$ water molecules per $\mathrm{CD}$ dimer $)$. Distribution of the unique water molecules in the crystal over nine positions rendered their crystallographic modelling difficult and the final refinement included 7 water molecules per CD dimer, in reasonable agreement with the TGA estimate. The final two-step mass loss of $7.6 \pm$ $0.5 \%$ in the temperature range $100-270{ }^{\circ} \mathrm{C}$ corresponds to release of the guest molecule from the $\mathrm{CD}$ (calcd. for a 2:1 host guest stoichiometry 7.2\%). There is no mass loss in the TG curve corresponding to the small endotherm B on the DSC trace. This endotherm (onset $\sim 120{ }^{\circ} \mathrm{C}$ ), is interpreted as being associated with initial rearrangement of the solid complex to yield a modified complex phase. PXRD patterns were acquired at different temperatures (Figure 9). In keeping with the above TGA/DSC interpretation, we note that the PXRD trace recorded at 110 ${ }^{\circ} \mathrm{C}$ shows some significant differences from that recorded at $40{ }^{\circ} \mathrm{C}$ (the effect of the dehydration process), while another significant change in the PXRD pattern occurs between 110 and $160{ }^{\circ} \mathrm{C}$ (accounting for the interpretation of the DSC event B).

As noted from the X-ray data, at low temperatures the cycluron molecule is fully encapsulated within the TRIMEA dimer and a significant change in structure (presumably including opening of the host primary 'lids' as an initial step) is necessary to liberate the guest molecules. The sharp endotherm $\mathrm{C}$, immediately followed by an exotherm [Figure 8(a)] is attributed to successive melting of the anhydrous complex and its rapid recrystallization to a new phase that subsequently releases the guest, yielding a final solid phase that melts at $\sim 200{ }^{\circ} \mathrm{C}$ 
(endotherm D). The variable-temperature PXRD data (Figure 9) again support the above interpretation of the DSC features in that the PXRD pattern at $160{ }^{\circ} \mathrm{C}$ does not match that of the single known crystalline form of the host TRIMEA, ${ }^{9}$ whereas at $200{ }^{\circ} \mathrm{C}$, the trace is in good agreement with that of pure TRIMEA.

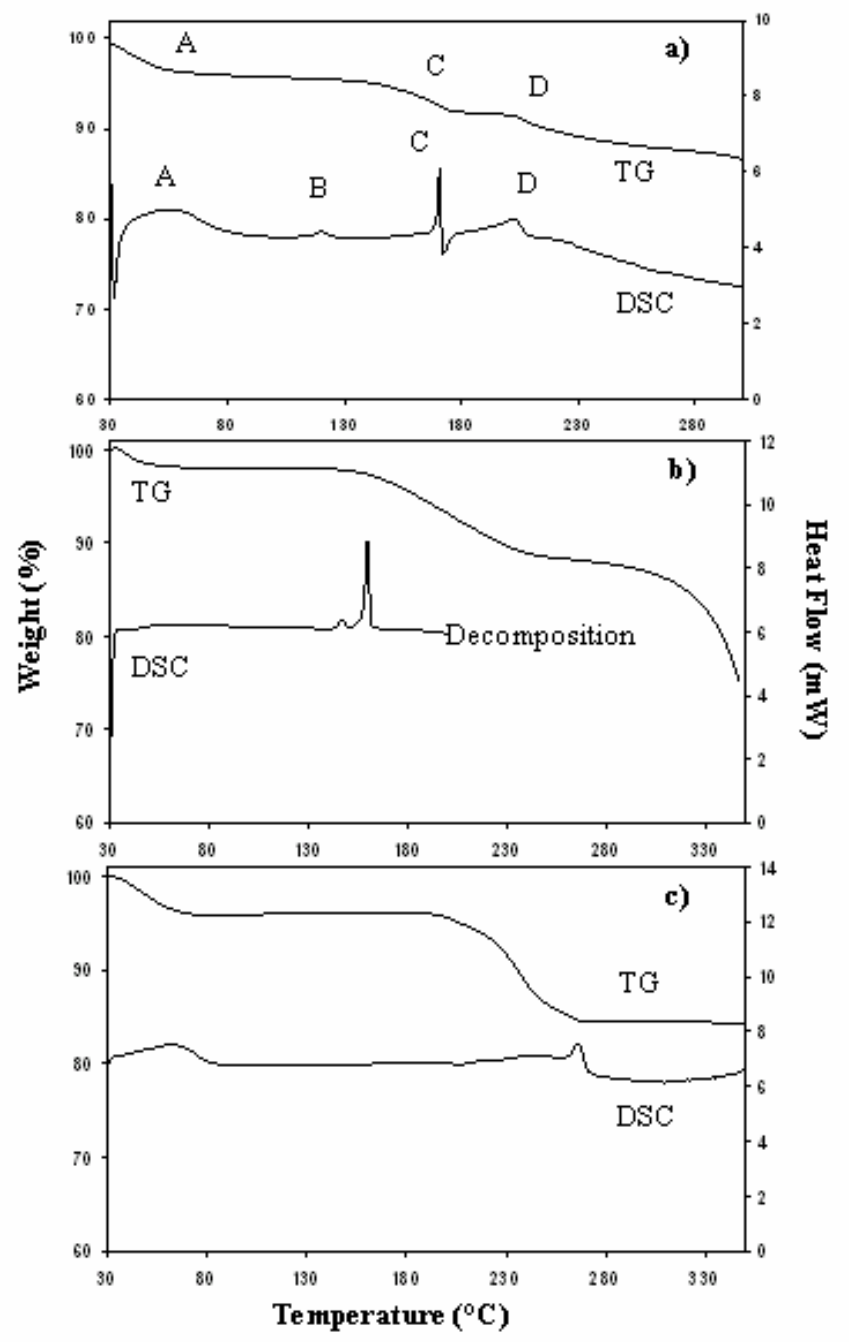

Figure 8. TGA and DSC data for complexes 1 (a), 2 (b) and 3 (c). The axis on the left refers to the TGA data while that on the right refers to the DSC data.

Complex (2). Crystals of TRIMEB cycluron $0.9 \mathrm{H}_{2} \mathrm{O}$ show an initial mass loss of $1.4 \pm 0.1 \%$ [ $\mathrm{n}=$ 2 , Figure $8(\mathrm{~b})]$. This is equivalent to $1.3 \pm 0.1$ water molecules per asymmetric unit. The crystal structure was modelled with 0.9 water molecules per host molecule. In this case, the somewhat higher experimental TGA mass loss can be attributed to the crystalline material that grows in a rosette formation, rendering surface-drying of the crystals prior to thermal analysis a difficult operation. The second mass loss of $10.5 \pm 0.1 \%(\mathrm{n}=11)$ measured from 130 to $280{ }^{\circ} \mathrm{C}$ is due to 
the guest molecule dissociating from the host (calcd. for 1:1 host-guest stoichiometry $12.1 \%$ ). The slightly lower TGA result for the host-guest stoichiometry is a consequence of the two events of melting and decomposition overlapping, i.e. occurring without a definite plateau between each process. The small endotherm on the DSC curve occurring at $148{ }^{\circ} \mathrm{C}$ may be due to rearrangement of the complex structure to one that is more favourable for guest loss. Following melting of the complex at $160^{\circ} \mathrm{C}$ (indicated by the large, sharp endotherm in the DSC trace), there is immediate loss of guest. The melting point lies in the range of reported melting points for other known TRIMEB inclusion complexes and differs from that of the known crystalline forms of TRIMEB itself $\left(148^{\circ} \mathrm{C}, 157^{\circ} \mathrm{C}\right) .{ }^{10}$

Complex (3). Figure 8(c) shows the TGA and DSC curves for DIMEB cycluron $2.9 \mathrm{H}_{2} \mathrm{O}$ (3). The TGA curve indicates an initial mass loss of $3.6 \pm 0.5 \%(\mathrm{n}=2)$, which is equivalent to $3.2 \pm 0.3$ water molecules per asymmetric unit. This is consistent with the number of water molecules included in the crystallographic asymmetric unit modelled in the X-ray analysis (2.9). Corresponding to this process, the DSC curve depicts an initial broad endotherm in the range $\sim 30-100{ }^{\circ} \mathrm{C}$. The second mass loss of $11.7 \%$ commencing at $\sim 180{ }^{\circ} \mathrm{C}$ is interpreted as the dissociation of the guest from the host (calcd. for 1:1 host-guest stoichiometry $12.5 \%$ ) and is reflected in DSC as the second broad endotherm spanning the range $200-280{ }^{\circ} \mathrm{C}$. The latter evidently comprises two distinct events (from the broad peak in the range $\sim 200-260{ }^{\circ} \mathrm{C}$ and a sharp endotherm peaking at $275^{\circ} \mathrm{C}$, as well as the change in the slope of the TGA trace at $\sim 245$ ${ }^{\circ} \mathrm{C}$ ). Host decomposition occurs beyond $330{ }^{\circ} \mathrm{C}$. The DSC trace for complex 3 is similar to those recorded for other DIMEB complexes containing organic guest molecules. ${ }^{11}$

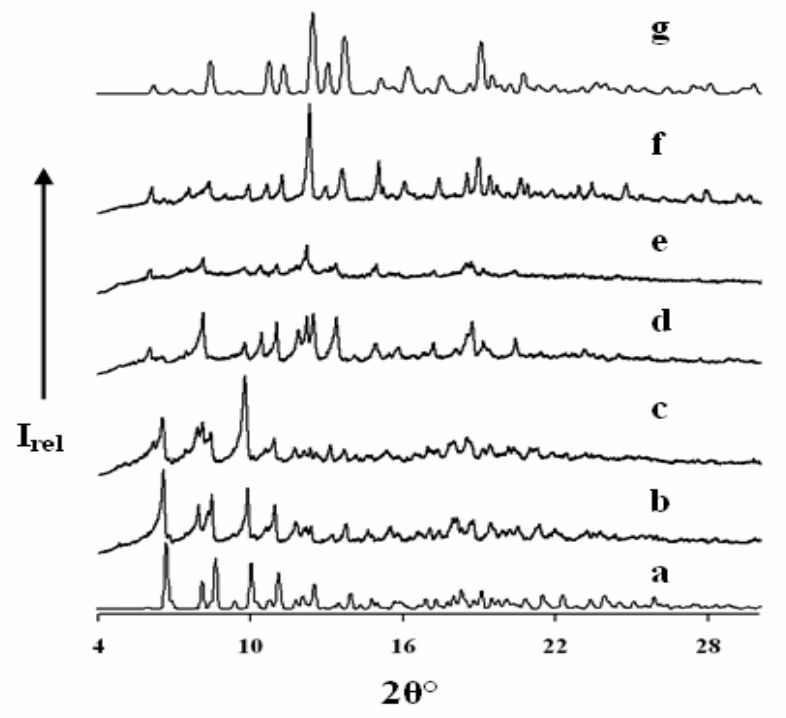

Figure 9. PXRD patterns: computed from the single crystal X-ray structure of complex 1 at -100 ${ }^{\circ} \mathrm{C}(\mathrm{a})$; experimental patterns at $40{ }^{\circ} \mathrm{C}(\mathrm{b}), 110^{\circ} \mathrm{C}$ (c), $160^{\circ} \mathrm{C}$ (d) and $200{ }^{\circ} \mathrm{C}(\mathrm{e})$; PXRD pattern recorded $24 \mathrm{~h}$ later at $25^{\circ} \mathrm{C}(\mathrm{f})$; computed PXRD pattern of the host TRIMEA (g).

In summary, the X-ray structural study confirms the affinity of the cycluron molecule for methylated CDs, revealing in addition a variety of guest inclusion modes accompanying 
variations in the $\mathrm{CD}$ ring size and methylation patterns. Host-guest interactions are primarily hydrophobic in nature and in none of the complexes is there significant interaction between the amide group and the $\mathrm{CD}$ cavity walls. In complexes $\mathbf{1}$ and $\mathbf{2}$, a water molecule donates a hydrogen bond to the $\mathrm{N}$ atom of the guest, but in complex $\mathbf{3}$ there are no guest-water interactions.

The guest cycluron molecule is released upon heating all three crystalline inclusion complexes. However, due to the significant differences in the chemical constitutions of the respective host molecules (TRIMEA, TRIMEB, DIMEB), the varying extents of guest encapsulation and the different crystal packing arrangements in 1-3, very distinctive thermal decomposition profiles are observed, the most complex behaviour being shown by the TRIMEA complex. Variable temperature PXRD was useful in interpreting the thermal events in the latter case. Inclusion of cycluron in DIMEB resulted in the highest level of guest thermal stabilization. This is attributed to the relative inflexibility of the DIMEB host molecule and cavity-entrapment of the cycluron molecule by blocking of both the primary and secondary sides of its parent $\mathrm{CD}$ by neighbouring host molecules.

\section{Experimental Section}

Materials and solid complex preparation. The host compounds hexakis(2,3,6-tri- $O$-methyl)- $\alpha$ CD (TRIMEA), heptakis(2,3,6-tri- $O$-methyl)- $\beta$-CD (TRIMEB) and heptakis(2,6-di- $O$-methyl)$\beta$-CD (DIMEB) were purchased from Cyclolab (Budapest, Hungary) with a purity $>98 \%$ for TRIMEB and DIMEB and $>97 \%$ for TRIMEA. Cycluron was purchased from Sigma Aldrich (USA) (Assay HPLC 99.9\%). These compounds were used as received.

Each inclusion complex was prepared using a 1:1 host-guest ratio. An aqueous saturated solution (total volume of $\sim 4 \mathrm{~mL}$ ) of each $\mathrm{CD}$ was prepared and an equimolar amount of cycluron was added with stirring at $20{ }^{\circ} \mathrm{C}$. The resulting clear solutions were filtered through a $0.45 \mu \mathrm{m}$ microfilter into a clean vial and placed in an oven at $65{ }^{\circ} \mathrm{C}$. Colourless single crystals of the (TRIMEA) $)_{2} \cdot$ cycluron $7 \mathrm{H}_{2} \mathrm{O} \quad$ 1, TRIMEB cycluron $\cdot 0.9 \mathrm{H}_{2} \mathrm{O} 2$ and DIMEB-cycluron $2.9 \mathrm{H}_{2} \mathrm{O} 3$ inclusion complexes were obtained within four to five days. Complex stoichiometry was determined by thermogravimetry which showed at least two distinctive mass losses for all three complexes. The water content in each crystal was determined from the first mass loss and the host-guest ratio was determined from the second (second and third in the case of $\mathbf{1}$ ), as described in the text.

\section{Thermal analysis}

Thermogravimetric analysis (TGA) and differential scanning calorimetric (DSC) measurements were performed using a Mettler Toledo TGA analyser and a Perkin-Elmer PC7-Series instrument respectively. Both instruments were operated with a constant heating rate of $10 \mathrm{~K} \mathrm{~min}^{-1}$ with dry nitrogen purge gas flowing at $30 \mathrm{~cm}^{3} \mathrm{~min}^{-1}$. The temperature range for each experiment was selected on the basis of the decomposition temperature of each CD. Samples in the range of 2-4 
mg were surface dried on filter paper and rapidly placed in an open alumina crucible for the TGA measurements. In the case of DSC measurements the surface dried sample was placed in closed vented aluminium pans.

\section{Powder X-ray diffraction}

A HUBER Imaging Plate Guinier Camera 670 was used with Ni-filtered $\mathrm{CuK} \alpha$-radiation $(\lambda=$ $1.5406 \AA$ ) generated by a Philips X-ray generator at $40 \mathrm{kV}$ and $25 \mathrm{~mA}$. The complex crystals were either applied directly onto Mylar ${ }^{\mathbb{B}}$ film or placed in a capillary and exposed to the radiation for 30 min with 10 multiscans to collect the data. A Huber High-Temperature Controller HTC 9634 unit was used with a capillary rotation device in order to obtain powder Xray diffraction (PXRD) patterns at elevated temperatures.

Experimental PXRD traces for 1-3 (not shown) were in very good agreement with the corresponding traces computed from the singe crystal XRD data, indicating that crystal selected for structural elucidation was in each case representative of the bulk material.

\section{Single crystal $\mathrm{X}$-ray diffraction}

Intensity data-collections for all three complexes were measured on a Nonius Kappa CCD diffractometer from crystals coated with Paratone $\mathrm{N}$ oil (Exxon Chemical Co., TX, USA). Complex crystals 1 and 2 were cooled to $173 \mathrm{~K}$ and complex crystal 3 to $143 \mathrm{~K}$ in a stream of nitrogen vapour to enhance diffraction quality. Data collection (COLLECT software ${ }^{12}$ ) involved a combination of $\varphi$ - and $\omega$-scans of $0.5^{\circ}$ for complexes 1 and 2 and $1.0^{\circ}$ for complex 3 . The programs DENZO-SMN and SCALEPACK were used for unit cell refinement and data reduction. ${ }^{13}$ The structures of complexes $\mathbf{1}$ and $\mathbf{3}$ were solved by direct methods (program SHELXD ${ }^{14}$ ), while that of complex 2 was solved by using the rigid skeleton of the isostructural complex TRIMEB-p-iodophenol ${ }^{7}$ as a trial model. All remaining non-H atoms of the host and guest were located in successive difference electron density maps. The structures were refined by full-matrix least-squares using program SHELXH-97. ${ }^{15}$ In some cases atoms were found to be disordered over two positions and these were modelled by assigning site-occupancy factors (s.o.f.s) of $x$ and 1-x, with $x$ variable. For all three structures, the hydrogen atoms of the host and the methine, methylene and methyl protons of the guest were geometrically constrained to their parent atoms and refined with linked temperature factors. The amide hydrogen (H9) of the guest molecule was not geometrically constrained to an idealised position for complexes $\mathbf{1}$ and $\mathbf{2}$ as these hydrogen atoms were apparent in the difference Fourier maps and did not conform to the regular planar geometry but rather a slightly pyramidal arrangement. The disordered amide hydrogen atoms (H9A and H9B) of complex 3 were placed in idealised positions and refined with a $\mathrm{U}_{\text {iso }}$ value 1.2 times those of their respective parent nitrogen atoms (N9A, N9B). The eight carbon atoms of the cyclooctyl ring and three secondary methoxy groups (C7B1, C8B1 and C7B3) of the host for complex 1 were refined isotropically due to their high thermal motion. Two primary methoxy groups and one secondary methoxy group of complex $\mathbf{2}$ were found to be disordered over two positions and were refined isotropically. Three methoxy carbon atoms of the 
host showed high thermal motion and were also refined isotropically. For the guest molecule of complex 2 three carbon atoms of the cyclooctyl ring and the two methyl groups were refined isotropically. All other non-hydrogen atoms of complex 2 were refined anisotropically. In the case of complex 3 there are three primary methoxy groups on glucose residues G4, G5 and G6, which are each disordered over two positions. The ordered methoxy carbon atoms on the secondary and primary rim of the $\mathrm{CD}$ were also found to have high thermal parameters which resulted in their being refined isotropically as well. The guest of complex $\mathbf{3}$ is disordered over two positions with only one atom being shared by each disordered component. The $\mathrm{C}$-C bonds in the cyclooctyl rings in complexes 1 and 3 were refined with a constrained bond length of $1.52 \AA$ $(\sigma=0.01 \AA)$ but the C-C-C angles were allowed free variation during refinement. For all three structure refinements, the Flack parameter could not distinguish the absolute structure and the Friedel opposites were therefore merged (MERG 4 in SHELXH-9 $7^{15}$ ). However, the absolute structures were correctly assigned on the basis of the known configurations of the chiral host molecules.

\section{Acknowledgements}

We thank the University of Cape Town and the NRF (Pretoria) for research support.

\section{References}

1. Szejtli, J. Pure Appl. Chem. 2004, 76, 1825.

2. Duchene, D.; Wouessidjewe, D. Drug Dev. Ind. Pharm. 1990, 16, 175.

3. Morillo, E. Applications of Cyclodextrins in Agrochemistry. In Cyclodextrins and Their Complexes; Dodziuk H., Ed.; Wiley-VCH Vrlag GmBH \& Co. KGaA.: Weinheim, 2006; pp 459.

4. Tomlin, C. D. S., Ed., The Pesticide Manual, $11^{\text {th }}$ Edn., British Crop Protection Council: Farnham, UK, 1997.

5. Mic, M.; Cruickshank, D.; Turcu, I. J. Phys.: Conf. Ser. 2009, 012 011. Available online: http://iopscience.iop.org/1742-6596/182/1/012011.

6. Cruickshank, D.; Rougier, N. M.; Vico, R. V.; de Rossi, R. H.; Buján, E. I.; Bourne, S. A.; Caira, M. R. Carbohydr. Res. 2010, 345, 141.

7. Harata, K.; Uekama, K.; Otagiri, M.; Hirayama, F. J. Inclusion Phenom. Mol. Recog. Chem. 1984, 1, 279.

8. Evans, D. G.; Boeyens, J. C. A. Acta Cryst. 1988, B44, 663.

9. Steiner, T.; Saenger, W. Carbohydr. Res. 1996, $282,53$.

10. Caira, M. R.; Bourne, S. A.; Mhlongo, W. T.; Dean, P. M. Chem. Commun. 2004, 2216.

11. de Vries, E. J. C.; Caira, M. R. Carbohydr. Res. 2008, 343, 2433. 
12. Hooft, R. COLLECT; Nonius BV: The Netherlands, 1998.

13. Otwinowski, Z.; Minor, W. In Processing of X-ray diffraction Data in Oscillation Mode in Methods in Enzymology; Carter, C. W.; Sweet, R. M., Eds.; Academic Press: New York, 1996; Vol. 276, pp 307.

14. Sheldrick, G. M. In Direct Methods for Solving Macromolecular Structures; Fortier, S., Ed.; Kluwer Academic Publishers: Dordrecht, 1998; pp 401.

15. Sheldrick, G. M. Acta Crystallogr., Sect. A, 2008, 64, 112. 